Experimental designs for neuroimaging - 1

Subtraction and beyond: The logic of experimental designs for neuroimaging

Russell A. Poldrack

Department of Psychology, Department of Psychiatry \& Biobehavioral Sciences, and Brain Research Institute, UCLA, Los Angeles, CA 90095-1563 
The goal of functional neuroimaging studies is generally to determine which particular brain regions or systems exhibit altered activity in response to the engagement of particular cognitive, emotional, or sensory processes. In order to determine these mappings between mental and neural processes, experimental designs are employed that attempt to manipulate a particular process and then examine how brain activity is affected by the manipulation. The goal of this chapter is to characterize the various forms of experimental design that have been used for functional neuroimaging, with a particular eye to how these designs constrain the inferences that can be supported.

When the neuroimaging researcher uses a task manipulation, he or she is (either implicitly or explicitly) assuming that the task can be decomposed into specific processes that can be independently manipulated. If the assumed decomposition is correct, then differences in brain activity can be mapped onto specific mental processes. This assumption of the decomposability of mental processes is shared with cognitive psychologists, who examine the effects of similar task manipulations on such behavioral variables as response times and task errors. Thus, both neuroimaging and cognitive psychology rely critically upon the assumption that mental processes can be functionally decomposed; this is a fundamental tenet of cognitive psychology that differentiates it from classical behaviorism. A full discussion of the status of mental decomposition is beyond the scope of the present paper; here I will take the decomposability of mental processes as a given, and refer the interested reader to some previous critiques (e.g., Skinner, 1963; Uttal, 2000) and rejoinders (e.g., Bechtel, 2002)

\section{The subtraction method}

The most basic, and still most commonly used, experimental design for neuroimaging 
is the subtraction method (see Figure 1). At least two conditions are presented, which putatively differ in the presence of a single cognitive process. For example, Petersen et al. (1988) presented subjects with nouns and asked them to either read the word aloud or generate a verb associated with the noun. The subtraction between these two conditions was meant to isolate the processes of semantic retrieval, under the assumption that all other processes (e.g., those involved in word recognition, speech production, breathing, etc.) remain constant between the two tasks. This is known as the "pure insertion" (PI) assumption, meaning that a single cognitive process can be inserted without affecting the remainder; this concept was first introduced by Donders (1868/1969) in the context of reaction times (cf. Sternberg, 1969). Subtraction remains the most common experimental design in cognitive neuroimaging studies because of its simplicity.

The core problem. This simplicity, however, is misleading: The PI assumption is simply not tenable in many situations. In neuroimaging, the PI assumption actually comprises two parallel assumptions, one regarding the insertability of cognitive processes (i.e., the same assumption made in response time studies), and a second regarding the insertability of neural processes. The assumption regarding cognitive insertability can potentially be tested using an approach known as the additive factors logic (Sternberg, 1969), in which one manipulates multiple factors in order to test for interactions between putatively separable functions. However, such tests of the PI assumption are only rarely presented in neuroimaging papers. In addition, the additive factors approach requires the strong assumption that transfer of information between processing stages occurs in a set of serial and discrete steps. The strongly interactive and recurrent nature of neural processing and prevalence of feedback connections between regions suggests that this 
assumption is incorrect. In addition, there is specific evidence for partial information transfer between stages of processing occurring in different brain regions from single-unit recordings (Bichot et al., 2001) and human electrophysiology (Miller and Hackley, 1992). These violations of the discrete stage assumption make it impossible to determine using additive factors logic whether different systems are additive or interactive (McClelland, 1979).

As pointed out by Friston et al. (1996), it is perfectly possible that a design could satisfy the PI assumption at the cognitive level yet fail to satisfy it at the neural level, and failure at either of these levels compromises any inferences based on the subtraction. In fact, the highly interactive and nonlinear nature of neural processing makes it very likely that this assumption will fail. Jennings et al. (1997) provided evidence of just such a failure. Subjects in their study performed judgments regarding semantics (deciding whether the object was a living thing) or letters (deciding whether the word had an "a"), under three different response conditions (spoken response, silent response, or mouse movement). Behavioral data revealed no interaction between task and response type for memory encoding (i.e., memory performance was similarly affected by the task across different response types), but there was a substantial interaction in the neural response as measured by PET: There was a much larger difference between encoding tasks in the left inferior prefrontal cortex under mouse response conditions than under mental response conditions, with spoken response falling in between. These data suggest that, even if the assumptions of the additive factors logic are tenable, demonstrating additivity at the level of behavior may not be sufficient to ensure the PI assumption of the subtractive method. If the PI assumption fails, then there is simply no way to determine what cognitive 
processes are reflected in the activation observed in the subtraction experiment.

The need for task analysis. A further problem with subtraction designs lies in how task comparisons are designed. A number of authors have noted (Sergent et al., 1992; Poeppel, 1996; Sartori and Umilta, 2000) that cognitive subtraction requires a formal task analysis in order to determine which particular cognitive process is being isolated by a subtraction. Such an analysis lays out the processes involved in performing the task, which is equivalent to a theory of how the task is performed. Unfortunately, such task analyses are very rarely presented in neuroimaging papers. Whereas formal theories from cognitive psychology could often provide substantial guidance as to the design of such tasks, it is uncommon for neuroimaging studies to take meaningful guidance from such theories. Rather, the task comparisons in many studies are based on intuitive judgments regarding the cognitive processes engaged by a particular task.

As an example, Poldrack et al. (1999) set out to isolate semantic and phonological processing in separate task comparisons. To do this, we presented subjects with four tasks: abstract/concrete judgments, syllable-counting judgments (with either words or pseudowords), and uppercase/lowercase judgments. The comparison of most interest was that between abstract/concrete judgments and syllable-counting judgments, which was meant to isolate processes specific to semantic retrieval (positive differences) or phonological manipulation (negative differences). However, no formal task analysis was presented to motivate these tasks (indeed, they were adopted because they had been previously used by other experimenters, who also did not present formal task analyses). An assessment of these tasks with regard to psycholinguistic theories of phonological or semantic retrieval reveals a number of difficulties in interpreting the findings. First, 
syllable-counting judgments with written materials actually require a number of different processes that might fall under the umbrella of "phonological processing": retrieval and/or assembly of phonological output forms, syllabification, metalinguistic counting of the syllable units, and working memory for phonological and metalinguistic information. Likewise, the semantic task may involve lexical semantic access, resolution of lexical ambiguity, retrieval of specific semantic cues in service of the decision, and working memory for this semantic and metalinguistic information. Thus, activation for subtractions between these tasks could reflect any of a number of theoretically distinct processes that differ between them. Together these points suggest that the conclusions deriving from many imaging studies using subtraction designs may provide a relatively coarse mapping of cognitive processes to brain function; to the degree that one wishes to map cognitive processes to brain regions as specifically as possible, then this is problematic.

A defense of subtraction. In opposition to arguments against subtraction, Petersen et al. (1998) argued that:

...confusion results from the lack of appreciation for the distinction between "cognitive subtraction" as an experimental design and interpretive strategy, and image subtraction as an analysis methodology. As seen above, cognitive subtraction can be used to design and interpret an imaging study, and imaging subtraction can be used to mirror the cognitive strategy. However, image subtraction does not make the assumption of pure insertion: experimental designs, analysis choices, and interpretive strategies do. Image subtraction is performed in part to mirror experimental design strategy, but more importantly it is done to 
reveal the differences in the hemodynamic signal between two conditions by subtracting the large amplitude complex anatomical background present in hemodynamic images. (p. 854)

It is certainly true that the computation of a subtraction between images does not require any assumptions about the underlying processes; however, most cognitive neuroscientists are not interested in creating subtraction images for the sake of making pretty pictures. Rather, they do this with the goal of mapping the observed subtraction results onto the mental processes that were manipulated in the study. This argument thus fails to salvage the subtraction approach.

Petersen et al. (1998) also argued that the assumption of pure insertion can be tested if the appropriate control conditions are performed. They focused on a particular kind of violation of pure insertion, wherein a process is engaged during the baseline task but not in the experimental task (e.g., daydreaming during a resting baseline). In order to assess the assumption of pure insertion, they compared activation during both a baseline task (word reading) and an experimental task (verb generation) with a resting baseline. Most regions showed a pattern that they took to confirm the PI assumption, with no difference between word reading and rest but a significant activation for verb generation versus reading; however, at least one region (in the posterior insula) showed activation for word reading versus rest but deactivation for word reading versus verb generation. This approach is interesting and does provide some added confidence in the results of a subtraction analysis, but it cannot unequivocally save the PI assumption. In particular, it makes a fairly strong assumption that it is possible to find some kind of ultimate neutral baseline against which to compare all other tasks (in this case, rest). However, there is a 
growing realization that all baseline conditions for fMRI are arbitrary, and in particular that resting is associated with engagement of specific mental processes (Binder et al., 1999; Stark and Squire, 2001).

Confounds and subtraction. One of the most common problems with subtraction designs is the presence of behavioral confounds between conditions. In nearly every case where two tasks are compared in a subtraction design, the task of interest is more difficult (as defined by longer reaction times or lower accuracy) than the baseline task. Given that the duration of neural firing is directly related to the resulting BOLD signal, this results in a difficulty for interpretation: Is the difference in activation due to the differential engagement of a particular process, or is it due to engagement of that process for different durations? This distinction is often taken to be rather esoteric in the neuroimaging field, but it is actually fundamental. For example, imagine a study that compared social versus non-social judgments about faces, in which the latter are performed more quickly than the former. A difference in activation is observed in a particular brain region, and this difference is attributed to social processes. However, it is equally possible that the region showing the difference in activation is actually related to motor programming, and that the more protracted response process in the social task results in more activation in the region. A number of studies using subtraction have dealt with this issue by using multiple baseline tasks that vary in their difficulty (e.g., Demb et al., 1995); this approach provides greater confidence that the resulting differences in activation are not driven by confounds.

The subtraction approach: what can we infer? The foregoing arguments provide strong reasons to distrust subtraction as a means of mapping cognitive processes onto 
neural activity. However, the approach remains quite prevalent in many parts of the functional imaging literature. It is striking that many subtraction studies find a common set of brain regions that are activated regardless of the specific task (Duncan and Owen, 2000), as well as a set of regions that are commonly deactivated relative to a baseline task (known as "task-independent decreases": e.g., Gusnard et al., 2001). One interpretation of this set of findings is that they reflect the effort/executive control confounds mentioned above, which are nearly always present in subtraction studies. Without the use of multiple baselines that vary in difficulty, it is nearly impossible to determine the specificity of activation in a subtraction design. Based on these concerns, it appears that simple subtraction designs are unable to establish reliable links between cognitive processes and neural structures.

\section{Alternatives to subtraction}

In order to sidestep the problems inherent in simple subtraction designs, a number of other fMRI design approaches have been developed (for other discussions of these approaches, see Friston et al., 1997; Aguirre and D'Esposito, 1999). Most of these methods do appear to avoid some of the criticisms of subtraction methods. However, closer examination reveals that they are subject either to many of the same fundamental assumptions as the subtraction approach, or to different but equally stringent assumptions regarding the relationship between task manipulations and the underlying cognitive processes.

Conjunction analysis. Price and Friston (1997) recommended an approach called "cognitive conjuction," which they suggested could circumvent the assumption of pure insertion. In the conjunction approach, subjects are presented with at least two different 
task subtractions, wherein only one putative cognitive process is shared between the different subtractions (see Figure 1). For example, one might examine phonological processing by conjoining two task comparisons: rhyme generation versus word naming (which isolates phonological processing operations) and syllable-counting versus silent reading (which isolates a large number of word recognition processes along with phonological processing). In the original version of this analysis (Price \& Friston, 1997), a significant conjunction required that each of the different subtractions show activation, and that there were no significant differences between the subtractions. Price and Friston (1997) argued that this version of conjunction analysis does not require the PI assumption, because regions showing differences across subtractions are by definition excluded. By contrast, the subtraction approach explicitly assumes the lack of interaction, and thus will be invalid in the presence of such interactions. In more recent versions (Friston et al., 1999), this approach was changed so that the conjunction simply finds regions that are commonly active at a particular threshold without regard to differences across subtractions; this version abandons the goal of discounting regions showing interactions, and thus is no more immune to the effects of interactions than standard subtraction analyses (cf. Caplan and Moo, 2004).

Another requirement of the conjunction approach, shared with the subtraction approach, is the need for a detailed task analysis in order to determine which components are subtracted in each comparison. In particular, conjunction analysis requires that only a single process is common between the conjoined subtractions. Take the previous example of phonological processing: It is quite difficult to ensure that phonological processing is the only common process between these two subtractions. For example, both rhyme 
generation vs. naming and syllable-counting vs. rest comparisons involve differential working memory demands, as well as differences in overall task difficulty which could differently engage task monitoring and effort mechanisms. As Caplan and Moo (2004) have pointed out, another problem is that subjects may implicitly process stimuli in ways that are not required by the task; for example, if a subject is asked to simply name a word, they may also automatically retrieve semantic knowledge about that word. These problems suggest that conjunction analysis may not generally overcome the fundamental limitations of the underlying subtractions. However, in comparison with simple subtraction it does offer some degree of additional confidence in putative mappings of cognitive processes to brain activity.

Factorial designs. Factorial designs involve the simultaneous manipulation of multiple experimental factors (see Figure 2). In such a design, the data are analyzed using an analysis of variance approach, which allows measurement of both main effects (which are equivalent to subtractions) and interactions, which occur when the effect of one factor is modulated by the level of another manipulated variable. For example, Gläscher et al. (2004) examined the interaction between facial identity and facial expression in order to test whether the fusiform gyrus and amygdala showed different patterns of interaction between these factors. Activity in the amygdala showed one pattern of interaction, wherein activity was greater when consistently fearful expressions were seen across a number of different faces, compared to all other combinations of identity and affect. The fusiform gyrus showed a different pattern of interaction, such that constant images (where the identity and emotion were held constant) showed less activity than any of the other conditions, which were all equal. 
These results show how factorial designs can be used to identify the ways in which specific factors interact. Rather than globally assuming pure insertion, it is possible to directly test whether the effects of one cognitive manipulation are modulated by other factors. The finding of such interactions provides additional insights into the mapping of cognitive processes to neural systems, by showing whether putatively distinct cognitive processes may converge or interact at the neural level. However, factorial designs still require the PI assumption, because each particular factor is assumed to isolate a specific cognitive process (cf. Aguirre \& D’Esposito, 1999); tests of the additivity of processes are possible for the manipulated processes but PI must be assumed for all other processes. For example, in the foregoing example, interpretation of the results requires the assumption that manipulations of facial expression do not cause changes in other factors, such as attentional arousal.

Parametric designs. Parametric designs involve the manipulation of a particular task parameter across multiple levels rather than comparison between different tasks (see Figure 3). This strategy has been used extensively in studies of the visual system, in which there are well-known relations between psychophysical parameters (e.g., stimulus contrast) and neural activity (e.g., Wandell, 1999). Similarly, studies of working memory have used a task paradigm known as "n-back", in which subjects are presented with a stream of stimuli (such as letters), and are asked determine whether the present stimulus matches the one presented $\mathrm{N}$ trials back. The value of $\mathrm{N}$ is manipulated, with 1-back being relatively easy, 2-back more difficult, and more than 3-back being impossible for many subjects; a "0-back" condition is often implemented by asking the subject to match each stimulus to a predefined target. Studies using this task have shown parametric 
increases in activation as $\mathrm{N}$ increases (e.g., Braver et al., 1997). With sufficient levels of the parameter, parametric designs also allow the estimation of nonlinear effects; for example, some parametric studies of the "n-back" task have shown an inverted-U function relating activation to working memory demands (e.g., Callicott et al., 1999). The inability of simple subtraction designs to find such nonlinear effects may underlie the conflicting results that are often seen in studies comparing patient (e.g., schizophrenia) and control groups; patients could show either greater or lesser activation than controls (or no difference) depending upon where in the function the two groups fall.

Because they involve the modulation of a single process rather than the presence/absence of that process, parametric designs theoretically avoid the pure insertion assumption. However, they replace it with what might be called the "pure modulation" assumption, which is the assumption that the manipulation affects only the degree to which the process is engaged and not the engagement/disengagement of any other processes. This assumption may fail in cases where changes in the parameter cause the task to be performed differently, or drive the engagement of additional processes. For example, if working memory load is increased parametrically to a level beyond the subject's capacity, then additional processes will be engaged at the point where memory fails (e.g., processes related to frustration or error detection). Parametric designs also require a task decomposition to understand exactly what process is being parametrically manipulated by the task. For example, increased working memory load increases the load on processes involved in memory maintenance, but may also increase the need for monitoring processes. One particular concern is that parametric manipulations are often correlated with task difficulty and response time. 
Priming/adaptation designs. Another class of designs, referred to as either repetition priming (e.g., Squire et al., 1992; Demb et al., 1995) or fMRI-adaptation (Grill-Spector et al., 1999; Grill-Spector and Malach, 2001) designs, take advantage of the fact that net activity in task-relevant regions often decreases when items are repeated in a task. In priming designs, regions showing repetition-related decreases in activity are inferred to process those stimulus properties that are repeated. Because the comparison is between two instances of exactly the same task, there is no need for the PI assumption. However, priming/adaptation designs require the assumption that the cognitive processes engaged during performance change only quantitatively and not qualitatively, similar to the "pure modulation" assumption for parametric designs. Given that some theories have proposed that repetition priming involves a change in processes rather than diminution in a single process (e.g., Logan, 1990), this issue is of potential concern. In addition, this approach requires a task analysis in order to understand the cognitive processes that are changed by repetition, in order to associate these processes with the regions of neural change. It should also be noted that repetition is almost invariably associated with decreased reaction time, and thus it is necessary to rule out whether observed decreases in activation reflect the consequences rather than the causes of the repetition effect.

It has been argued that adaptation-fMRI designs can provide better resolution of neural representations than direct comparisons between conditions (Grill-Spector et al., 1999, 2001). In this approach, a stimulus is repeatedly presented, thus putatively inducing adaptation in those neurons that respond selectively to that stimulus. Then, some feature of the stimulus is changed (e.g., size of an object). Regions showing release from adaptation are inferred to code for the particular feature that was changed, because 
neurons that are invariant to the altered feature should treat the stimulus exactly like the previous repetition and thus not show a release from adaptation. The adaptation method may be more powerful than simple categorical comparison of different stimulus types, because it equates everything except for the processing history of the stimulus and thus avoids some of the potential confounds inherent in comparing different items (cf. Henson, 2003). However, it may be invalid to interpret these changes in terms of the local representation in the regions showing adaptation. It is more likely that they reflect representations in upstream regions (where the changes in spike rate actually occur). Furthermore, there is evidence from single-unit recording that stimulus selectivity as inferred from adaptation responses may differ from the selectivity of the neuron's response to novel stimuli (Sawamura et al., 2006).

\section{Blocked vs. event-related designs and behaviorally-driven comparisons}

Somewhat orthogonal to the experimental design distinctions outlined above is the distinction between blocked and event-related fMRI designs. In a blocked design, subjects perform the tasks of interest for alternating blocks of time (generally lasting about 16-40 seconds), and the statistical model treats the entire block as a single entity (e.g., modeling the task design using a boxcar function). In an event-related design, trials of various types or tasks are presented in a pseudorandom order, with periods of rest or visual fixation interspersed between trials (Buckner et al., 1996; Wagner et al., 1998), and trials are modeled individually. Event-related designs have been embraced because they may be more psychologically realistic than blocked designs by making events less predictable. For example, in a study of recognition memory designed to compare activity evoked by old versus new stimuli, the presentation of long, predictable blocks of old or 
new stimuli may result in undesirable strategic effects. Event-related designs are also useful because they allow the sorting of trials based on the subject's behavior. For example, Brewer et al. (1998) presented subjects with pictures to study, and then tested their later memory for those pictures. The trial-by-trial fMRI data acquired during memory encoding were sorted depending upon whether the subject subsequently remembered or forgot each item, which revealed several regions whose activity predicted later memory. This approach is very useful because it relies upon actual behavior rather than the putative effects of a task manipulation.

Despite the power of event-related designs, there are cautions that must be appreciated. First, the use of behaviorally-defined comparisons, rather than task comparisons, does not eliminate the need for a decomposition of the cognitive processes revealed by the comparison. The finding that behavioral differences are associated with differences in brain activity is not particularly enlightening, unless it provides insights into how the cognitive processes underlying that behavioral difference are implemented in the brain. For example, studies of memory retrieval have compared activity on different classes of trials sorted by behavioral outcomes, such as successfully remembered items ("hits") versus forgotten items (“misses") (e.g., Eldridge et al., 2000). When activity is found in particular regions in relation to memory outcomes in particular brain areas (e.g., hippocampus), it has been associated with particular aspects of mnemonic experience. This conclusion may be well founded, particularly given everything else that is known about the role of the hippocampus is memory, but it is also possible that such differences could reflect other cognitive processes (e.g., processes related more generally to success on a task). It is thus necessary to decompose 
behaviorally-driven comparisons in the same way as task-driven comparisons.

Second, it is sometimes incorrectly assumed that event-related fMRI does not require the same subtraction assumptions as blocked designs. This illusion may stem from the fact that event-related fMRI allows one to estimate the hemodynamic response that is evoked by a particular trial condition; what is sometimes overlooked is the fact that this response is estimated against a particular baseline, generally a "null event" such as visual fixation or resting state. In this sense, inferences from event-related fMRI are no different from those based on blocked designs with regard to the logical nature of the comparisons.

\section{Conclusions}

This chapter has outlined the primary methods used for experimental designs in neuroimaging. In response to the well-known problems of the subtraction method, a number of approaches have been proposed. However, none of these approaches eliminates the need for an understanding of how the task manipulation relates to the underlying cognitive processes, because in each case intepretation of the results relies upon knowing exactly what processes have been modulated. Although the parametric and priming approaches do not require the PI assumption, they instead require a "pure modulation" assumption that may be equally as tenuous as the PI assumption. Eventrelated fMRI designs do not alleviate the need for any of these assumptions. Furthermore, in any fMRI design, an understanding of how neural activity relates to cognitive processes requires that the task be decomposed into particular processes, in essence requiring a theory of how the task is performed.

The less charitable reader might be tempted at this point to ask why the appropriate 
conclusion from the foregoing review is not to give up on fMRI as a means to understand the relation between cognitive and neural processes. Before addressing this specifically in the context of fMRI, it should first be noted that the project of cognitive psychology suffers from many of the same conceptual difficulties regarding the mapping of cognitive processes onto data (which in the case of cognitive psychology comprises data regarding behavior rather than brain imaging data). Not surprisingly, the harshest criticisms of neuroimaging have come from theorists who more fundamentally reject the decomposition of cognitive processes, either from behaviorists (Uttal, 2000) or dynamic systems theorists (van Orden and Papp, 1997). Thus, giving up on fMRI for these reasons would entail a more general abandonment of the goal of a mechanistic theory of mental processes.

Regarding fMRI more specifically, there are glimmers of hope amidst the sea of futile subtractions. One source of optimism comes from a number of recent meta-analyses of neuroimaging data (e.g., Turkeltaub et al., 2002; Wager and Smith, 2003; Owen et al., 2005; Nee et al., 2007). These analyses have demonstrated a strong degree of consistency in associations between classes of task manipulations and activation patterns. These findings can be viewed as a "conceptual conjunction analysis," showing that specific regions are consistently associated with particular brain regions even when other factors are varied across studies. The finding of consistency does not salvage the pure insertion assumption, but it does suggest that whatever processes are being mapped in subtraction studies are reliably associated with particular brain structures.

More important than consistency is specificity, wherein particular patterns of brain activity are selectively associated with particular cognitive manipulations. Meta-analyses 
have shown some degree of specificity. However, particularly in higher-order association cortices, any particular region may be associated with a number of different cognitive manipulations, though there do seem to be broader-scale functional gradients across cortical regions. For example, more anterior regions in prefrontal cortex appear to code for higher-order relations compared to more posterior regions (cf. Koechlin et al., 2003). Further work will be necessary to determine to what degree this lack of specificity reflects problems with the current cognitive ontology versus true distribution of function across the cortex (Poldrack, 2006). If highly specific patterns of brain activity related to specific cognitive manipulations can be identified, then this would blunt (though not eliminate) some concerns about the possible problems with subtraction, by suggesting that whatever processes are being isolated by convergent subtractions are neurobiologically coherent.

Another source of optimism comes from the integration of computational modeling with functional neuroimaging. To the degree that a computational model reflects an explicit theory of the processes underlying a task, it provides a more principled way to relate brain activity to specific cognitive functions. There is a wide range of approaches to integrating computational modeling and neuroimaging, but most involve the use of computational models to create model-based regressors that are then used for analysis of fMRI data. For example, Brown and Braver (2005) compared the predictions of two different computational models in order to determine whether activity in the anterior cingulate was better explained by conflict detection or error likelihood prediction. Other approaches have used model comparisons to determine whether separate brain systems may be characterized by different computational functions. For example, Hampton et al. 
(2006) used two computational models that varied in their degree of structure (a simple reinforcement learning model and a more complex Markov model), and found that different brain systems showed patterns of activity that correlated with each of the different computational processes. Such analyses still involve comparison between imaging conditions, but the comparisons are based on quantitative outputs from a computational model rather than qualitative manipulations of task or stimulus conditions. Although they do not eliminate the need for assumptions regarding the relation between cognitive and neural processes (such as the pure modulation assumption of parametric designs), they do provide more confidence that brain activity is being mapped to processes that play a specific cognitive role by virtue of performing a particular computation. 


\section{Acknowledgments}

Preparation of this chapter was supported by grants from the National Science Foundation (DMI-0433693) and National Institutes of Health (P20 RR020750 to R. Bilder). Thanks to Martin Bunzl and Marisa Geoghegan for helpful comments on a earlier draft. 
Figure Captions.

Figure 1. A schematic of the subtraction design and conjunction analysis. Each row in each panel represents a particular cognitive task or subtraction, and the columns represent four different cognitive processes. The number one represents presence of that processes and zero represents absence for the specific task.

Figure 2. A schematic of a factorial design, in which two processes (P1 and P2) are factorially manipulated across four conditions (C1-C4). This allows separate testing of the main effects of either condition and the interaction between them.

Figure 3. A schematic of a parametric design. In this case, Process P2 is manipulated parametrically across the four task conditions $\mathrm{C} 1-\mathrm{C} 4$. 
References

Aguirre GK, D'Esposito M (1999) Experimental design for brain fMRI. In: Functional MRI (Moonen CTW, Bandettini PA, eds), pp 369-380. Berlin ; New York: Springer.

Bechtel W (2002) Decomposing the mind-brain: A long-term pursuit. Brain and Mind 3:229-242.

Bichot NP, Chenchal Rao S, Schall JD (2001) Continuous processing in macaque frontal cortex during visual search. Neuropsychologia 39:972-982.

Binder JR, Frost JA, Hammeke TA, Bellgowan PS, Rao SM, Cox RW (1999) Conceptual processing during the conscious resting state. A functional MRI study. J Cogn Neurosci 11:80-95.

Braver TS, Cohen JD, Nystrom LE, Jonides J, Smith EE, Noll DC (1997) A parametric study of prefrontal cortex involvement in human working memory. Neuroimage $5: 49-62$.

Brewer JB, Zhao Z, Desmond JE, Glover GH, Gabrieli JD (1998) Making memories: brain activity that predicts how well visual experience will be remembered [see comments]. Science 281:1185-1187.

Brown JW, Braver TS (2005) Learned predictions of error likelihood in the anterior cingulate cortex. Science 307:1118-1121.

Buckner RL, Bandettini PA, O'craven KM, Savoy RL, Petersen SE, Raichle ME, Rosen BR (1996) Detection of cortical activation during averaged single trials of a cognitive task using functional magnetic resonance imaging. Proceedings Of The 
National Academy Of Sciences Of The United States Of America 93:1487814883.

Callicott JH, Mattay VS, Bertolino A, Finn K, Coppola R, Frank JA, Goldberg TE, Weinberger DR (1999) Physiological characteristics of capacity constraints in working memory as revealed by functional MRI. Cereb Cortex 9:20-26.

Caplan D, Moo L (2004) Cognitive conjunction and cognitive functions. Neuroimage 21:751-756.

Demb JB, Desmond JE, Wagner AD, Vaidya CJ, Glover GH, Gabrieli JD (1995) Semantic encoding and retrieval in the left inferior prefrontal cortex: a functional MRI study of task difficulty and process specificity. J Neurosci 15:5870-5878.

Donders FC (1868/1969) On the speed of mental processes. (Translated by W. G. Koster). Acta Psychologica 30:412-431.

Duncan J, Owen AM (2000) Common regions of the human frontal lobe recruited by diverse cognitive demands. Trends Neurosci 23:475-483.

Eldridge LL, Knowlton BJ, Furmanski CS, Bookheimer SY, Engel SA (2000)

Remembering episodes: a selective role for the hippocampus during retrieval. Nat Neurosci 3:1149-1152.

Friston KJ, Price CJ, Buechel C, Frackowiak RSJ (1997) A taxonomy of study design. In: Human Brain Function (Frackowiak RSJ, Friston KJ, Frith CD, Dolan RJ, Mazziotta JC, eds), pp 141-162. San Diego: Academic Press.

Friston KJ, Holmes AP, Price CJ, Buchel C, Worsley KJ (1999) Multisubject fMRI studies and conjunction analyses. Neuroimage 10:385-396.

Friston KJ, Price CJ, Fletcher P, Moore C, Frackowiak RS, Dolan RJ (1996) The trouble 
with cognitive subtraction. Neuroimage 4:97-104.

Glascher J, Tuscher O, Weiller C, Buchel C (2004) Elevated responses to constant facial emotions in different faces in the human amygdala: an fMRI study of facial identity and expression. BMC Neurosci 5:45.

Grill-Spector K, Malach R (2001) fMR-adaptation: a tool for studying the functional properties of human cortical neurons. Acta Psychol (Amst) 107:293-321.

Grill-Spector K, Kushnir T, Edelman S, Avidan G, Itzchak Y, Malach R (1999) Differential processing of objects under various viewing conditions in the human lateral occipital complex. Neuron 24:187-203.

Gusnard DA, Raichle ME, Raichle ME (2001) Searching for a baseline: functional imaging and the resting human brain. Nat Rev Neurosci 2:685-694.

Jennings JM, McIntosh AR, Kapur S, Tulving E, Houle S (1997) Cognitive subtractions may not add up: the interaction between semantic processing and response mode. Neuroimage 5:229-239.

Koechlin E, Ody C, Kouneiher F (2003) The architecture of cognitive control in the human prefrontal cortex. Science 302:1181-1185.

Logan GD (1990) Repetition priming and automaticity: Common underlying mechanisms? Cognitive Psychology 22:1-35.

McClelland JL (1979) On the time relations of mental processes: An examination of systems in cascade. Psychological Review 86:287-330.

Miller J, Hackley SA (1992) Electrophysiological evidence for temporal overlap among contingent mental processes. J Exp Psychol Gen 121:195-209.

Nee DE, Wager TD, Jonides J (2007) Interference resolution: insights from a meta- 
analysis of neuroimaging tasks. Cogn Affect Behav Neurosci 7:1-17.

Owen AM, McMillan KM, Laird AR, Bullmore E (2005) N-back working memory paradigm: a meta-analysis of normative functional neuroimaging studies. Hum Brain Mapp 25:46-59.

Petersen SE, van Mier H, Fiez JA, Raichle ME (1998) The effects of practice on the functional anatomy of task performance. Proc Natl Acad Sci U S A 95:853-860.

Petersen SE, Fox PT, Posner MI, Mintun M, Raichle ME (1988) Positron emission tomographic studies of the cortical anatomy of single-word processing. Nature 331:585-589.

Poeppel D (1996) A critical review of PET studies of phonological processing. Brain Lang 55:317-351; discussion 352-385.

Poldrack RA (2006) Can cognitive processes be inferred from neuroimaging data? Trends Cogn Sci 10:59-63.

Poldrack RA, Wagner AD, Prull MW, Desmond JE, Glover GH, Gabrieli JDE (1999) Functional specialization for semantic and phonological processing in the left inferior frontal cortex. NeuroImage 10:15-35.

Price CJ, Friston KJ (1997) Cognitive conjunction: a new approach to brain activation experiments. Neuroimage 5:261-270.

Sartori G, Umilta C (2000) How to avoid the fallacies of cognitive subtraction in brain imaging. Brain Lang 74:191-212.

Sawamura H, Orban GA, Vogels R (2006) Selectivity of neuronal adaptation does not match response selectivity: a single-cell study of the FMRI adaptation paradigm. Neuron 49:307-318. 
Sergent J, Zuck E, Levesque M, MacDonald B (1992) Positron emission tomography study of letter and object processing: empirical findings and methodological considerations. Cereb Cortex 2:68-80.

Skinner BF (1963) Behaviorism at fifty. Science 140:951-958.

Squire LR, Ojemann JG, Miezin FM, Petersen SE, Videen TO, Raichle ME (1992)

Activation of the hippocampus in normal humans: A functional anatomical study of memory. Proc Natl Acad Sci U S A 89:1837-1841.

Stark CE, Squire LR (2001) When zero is not zero: the problem of ambiguous baseline conditions in fMRI. Proc Natl Acad Sci U S A 98:12760-12766.

Sternberg S (1969) The discovery of processing stages: Extension of Donders method. Acta Psychologica 30:276-315.

Turkeltaub PE, Eden GF, Jones KM, Zeffiro TA (2002) Meta-analysis of the functional neuroanatomy of single-word reading: method and validation. Neuroimage $16: 765-780$.

Uttal WR (2000) The war between mentalism and behaviorism. Mahwah, NJ: Erlbaum. van Orden CG, Papp KR (1997) Functional neuroimages fail to discover pieces of mind in the parts of the brain. Philosophy of Science 63.

Wager TD, Smith EE (2003) Neuroimaging studies of working memory: a meta-analysis. Cogn Affect Behav Neurosci 3:255-274.

Wagner AD, Schacter DL, Rotte M, Koutstaal W, Maril A, Dale AM, Rosen BR, Buckner RL (1998) Building memories: remembering and forgetting of verbal experiences as predicted by brain activity [see comments]. Science 281:11881191. 
Experimental designs for neuroimaging - 28

Wandell BA (1999) Computational neuroimaging of human visual cortex. Annu Rev

Neurosci 22:145-173. 
P1 P2 P3 P4

Task A

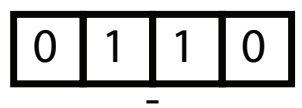

Task B

Subtraction
P1 P2 P3 P4

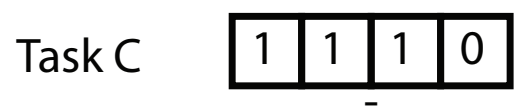
Task D

Subtraction \begin{tabular}{|l|l|l|l|}
\hline 0 & 1 & 0 & 0 \\
\hline
\end{tabular}

Conjunction \begin{tabular}{|l|l|l|l|}
\hline 0 & 1 & 0 & 0 \\
\hline
\end{tabular} 
P1 P2 P3 P4

C1 1 \begin{tabular}{|l|l|l|l|}
\hline 1 & 1 & 1 & 0 \\
\hline
\end{tabular}

Factorial manipulation

C2 \begin{tabular}{|l|l|l|l|}
\hline 1 & 0 & 1 & 0 \\
\hline
\end{tabular}

Main effect of P1: $(\mathrm{C} 1+\mathrm{C} 2)-(\mathrm{C} 3+\mathrm{C} 4)$ Main effect of P2: $(\mathrm{C} 1+\mathrm{C} 3)-(\mathrm{C} 2+\mathrm{C} 4)$ of processes

$\mathrm{P} 1$ and $\mathrm{P} 2$

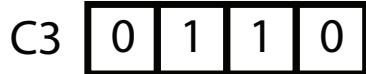
C4 40 \begin{tabular}{l|l|l|l|}
\hline 0 & 0 & 1 & 0 \\
\hline
\end{tabular} Interaction: $(\mathrm{C} 1+\mathrm{C} 4)-(\mathrm{C} 2+\mathrm{C} 3)$ 


\section{P1 P2 P3 P4 \\ C1 \begin{tabular}{|l|l|l|l|}
\hline 1 & .2 & 1 & 0 \\
\hline
\end{tabular}}

Parametric

manipulation

\begin{tabular}{lll|l|l|l|} 
of process P2 & C3 & 1 & .6 & 1 & 0 \\
\hline
\end{tabular}

C4 \begin{tabular}{|l|l|l|l|}
\hline 1 & .8 & 1 & 0 \\
\hline
\end{tabular} 